\title{
Optimising for Scale in Globally Multiply-Linked Gravitational Point Set Registration Leads to Singularities*
}

\author{
Vladislav Golyanik Christian Theobalt \\ Max Planck Institute for Informatics, Saarland Informatics Campus
}

\begin{abstract}
The recently introduced gravitational point set registration methods can robustly align point sets with high noise ratios. While many results with rotation and translation resolution have been presented in the literature so far, the uncertainty about the scale resolving capability of globally multiply-linked gravitational point set registration methods is remaining. To address the uncertainty, we analyse the gravitational potential energy (GPE) functional of the system with two point sets with multivariate calculus and come to the conclusion that GPE of a singularity, i.e., the state when the template collapses to a single point, always has a lower energy than the state of the optimal alignment. Moreover, the GPE as a function of scale monotonically increases and does not have equienergetic states, which we prove for several hollow and volumetric geometric primitives in $\mathbb{R}^{2}$ and $\mathbb{R}^{3}$ including a unit circle, a unit sphere, a unit disk and a unit ball. We perform a series of experiments with various regular shapes and different combinations of references and templates to validate our findings. The consequence is that in practice, it is highly unlikely for globally multiply-linked gravitational alignment to resolve the scale accurately. We propose ways to overcome the limitation in scale resolution, which can be considered in the next generation of gravitational approaches.
\end{abstract}

\section{Introduction}

Rigid point set registration is the problem of the recovery a rigid transformation - translation $\mathbf{t}$ and rotation $\mathbf{R}-$ aligning two or several point sets into the common reference frame. Often, template scaling $s$ is also included, resulting in seven degrees of freedom $(\mathrm{DoF})$ alignment $[21,20]$. In the case of two point sets, it is common to distinguish a fixed point set or a reference, and a point set being transformed, or a template.

One of the newly proposed methods for rigid point set registration is gravitational approach (GA) relying on simu-

\footnotetext{
* supported by the ERC Consolidator Grant 4DReply (770784)
}

lation of particle dynamics [14, 15]. GA aligns point sets by interpreting them as particle swarms interacting in a gravitational force field. Every particle possesses a mass condensed in an infinitesimal volume so that the sets can overlap. The optimality criterion in GA is locally minimal gravitational potential energy (GPE) $\mathbf{E}(\mathbf{R}, \mathbf{t})$ of the system, see Eq. (1) in Sec. 3.

In [14], GPE is optimised implicitly by updating the forces acting on the template particles. An accurate solution requires an additional dumping parameter and a careful parameter choice. Compared to several other methods such as iterative closest point (ICP) [4] and coherent point drift (CDP) [21], GA was shown to be more robust in the scenarios with high noise ratios in the data, not lastly due to the globally multiply-linked character of point interactions.

GA was claimed to be capable of scale recovery under global multiply-linking. Notwithstanding, Golyanik et al. [14] report that scale resolution is not reliable in GA and that shrinkage of the template to a single point can be witnessed if the parameters are chosen suboptimally. In GA for non-rigid alignment, Ali et al. [3] split point sets into multiple overlapping patches and update the local patch scales as curl-free components of the unconstrained displacement fields. Due to the fine-grained region interleaving and inter-patch regularisation for individual point displacements, patch scales are well regularised and change insignificantly during the alignment. Generally, non-rigid GA requires the point sets provided in the same scale (up to small deviations caused by deformations).

\subsection{Contributions}

All in all, gravitational methods [14, 2, 3] leave uncertainty concerning how well they can resolve scale. Currently, there is no study which systematically investigates scale resolution aspects of gravitational methods. To address this uncertainty, we analyse the GPE as a function of the scale $s$ with multivariate calculus for several geometric primitives in $\mathbb{R}^{2}$ and $\mathbb{R}^{3}$ including a unit circle, a unit sphere, a unit disk and a unit ball. The main contribution of this paper is a thorough mathematically principled analysis of scaling resolving capability of multiply-linked 
gravitational point set registration methods. One of the primary assumptions of GA is that the optimal alignment is attained in the state of locally minimal GPE. We show that when the template scaling is allowed, the locally optimal GPE assumption is not fulfiled. This explains the repeatedly reported difficulties of GA in scale resolution, including excessive parameter tuning and heuristics [14, 2, 3]. Additionally, we perform a series of experiments with different regular shapes and various combinations of references and templates to support our findings.

\subsection{Paper Structure}

The remainder of the paper is organised as follows. Next sections review related work in Sec. 2 and rigid GA in Sec. 3. Sec. 4 describes the proof methodology and assumptions. The derivations for regular surfaces and volumetric shapes are provided in Secs. 5 and 6, respectively, and the exposition is concluded in Secs. 7 and 8. Finally, Appendix A provides further details for the proofs.

\section{Related Work}

In the seminal work of Besl and McKay [4], rigid point set registration is solved by alternatingly updating correspondences between the point clouds and the rigid transformation relating point pairs. The correspondences are established according to the deterministic nearest-neighbour rule, which is eponymous for the method's name, i.e., iterative closest point (ICP). Thus, point set registration in ICP comes down to a series of transformation estimation problems. Although the classic ICP is a widely-used technique with multiple variants and improvements shown in the literature $[7,16,22,10]$, its disadvantages include high sensitivity to noise, suboptimal initialisations, clustered outliers and missing data, due to one-to-one point correspondences.

To alleviate the limitations of ICP, multiple successor approaches relax binary correspondences to soft correspondences [13] and align point sets probabilistically [8, 20]. In coherent point drift (CPD) [21], alignment is performed by expectation-maximisation for fitting template points interpreted as Gaussian mixture models (GMM) to the points of the reference. Kernel Correlation (KC) approach [24] reduces point set alignment to the minimisation of the Renyi's quadratic entropy of the joint template-reference system. In both methods [24, 21], points interact de facto in the locally multiply-linked manner (the influence attenuates rapidly).

Multiple methods relax the rigidity constraints and recover general displacement fields between a template and a reference while imposing spatial smoothness on the displacement fields [8, 20, 21, 3, 25, 23]. It is often assumed that the topology of template point sets cannot change, while non-isometric deformations including shrinkage and dilatations are allowed. Affine transformations including scaling and shearing constitute a special case of non-rigid deformations. Similarly to a rigid transformation, scaling and shearing are parametric transformations which can be expressed by a single matrix applied to all points simultaneously. Scaling and shearing do not preserve absolute distances between points as well as angles between surfaces and lines, but they preserve distance ratios on surfaces. Two prominent examples of rigid point set alignment approaches which can be generalised for scale resolution are CPD [21] and GMM registration (GMR) [20].

Since recently, physically inspired methods are enriching the arsenal of point set registration methods [9, 14, 2, 3, 19, 15]. Deng and coworkers cast point sets into the Schrödinger distance transform representation and align them by minimising a geodesic distance on a unit Hilbert sphere [9]. Golyanik et al. [14] and Ali et al. [3] interpret point sets as particle swarms moving in gravitational force fields. The state of the locally optimal alignment is achieved when the gravitational potential energy (GPE) of the system is locally minimal.

In the gravitational approach (GA) [14], all points of the template interact with all points of the reference. In other words, all reference points influence each template point simultaneously following the principle of force superposition. Such global multiply-linking is especially advantageous in rotation resolution and when handling point clouds with uniformly distributed noise [15]. GA was claimed to support scale, though at the same time, the authors explicitly indicate possible difficulties and instability of the scale resolution when the point sets are interacting in the globally multiply-linked manner [14]. In GA with a shape constraint [2], the scale is resolved in one shot by comparing the spatial extents of the point sets, disjointly from the translation and rotation estimation. The accuracy of this scale resolution method decreases if point sets contain clustered outliers or missing data. Another limitation is that such scale resolution does not allow to benefit from the enhanced robustness to noise and the entire paradigm of GA.

Currently, there is no systematic analysis of scale resolving capabilities of gravitational point set registration methods. None of the works on gravitational point set alignment analyses the reasons for the poor performance when the scale is included as a parameter in the optimisation. To fill this gap, in this paper we focus on the scale resolution of gravitational approaches. Compared to previous works, we provide a mathematically principled analysis of the GA energy landscape parametrised by scale.

\section{Particle Dynamics Based Approaches}

Newtonian Gravitational Approach [14]. If a system of two point sets $\{\mathbf{X}, \mathbf{Y}\}$ is given, whereby $\mathbf{X}$ is the fixed reference, the inputs can be aligned by minimising the mutual gravitational potential energy (GPE) $\mathbf{E}$ of the correspond- 
ing system of particles in the force field induced by $\mathbf{X}$ :

$$
\mathbf{E}(\mathbf{R}, \mathbf{t})=-G \sum_{i, j} \frac{m_{\mathbf{y}_{i}} m_{\mathbf{x}_{j}}}{\left\|\mathbf{R} \mathbf{y}_{i}+\mathbf{t}-\mathbf{x}_{j}\right\|_{2}+\epsilon},
$$

where $m_{\mathbf{y}_{i}}$ and $m_{\mathbf{x}_{j}}$ denote masses, $\left[\mathbf{y}_{i}\right]=\mathbf{Y},\left[\mathbf{x}_{j}\right]=\mathbf{X}, G$ is the gravitational constant and $\epsilon$ is softening length which regularises too close encounters of the particles. In [14], this energy is minimised implicitly by updating the forces $\overrightarrow{\mathbf{f}}_{i}$ acting on particles $\mathbf{y}_{i}$, accelerations, velocities $v_{i}^{t+1}$ and individual displacements $d_{i}^{t+1}$ - according to Newton's second law of motion [1]:

$$
\overrightarrow{\mathbf{f}}_{i}=-G m_{\mathbf{y}_{i}} \sum_{j} m_{\mathbf{x}_{j}}\left(\left\|\mathbf{y}_{i}-\mathbf{x}_{j}\right\|^{2}+\epsilon^{2}\right)^{-3 / 2} \hat{\mathbf{n}}_{i, j}-\eta v_{i}^{t},
$$

with $\eta$ denoting the strength of the per-particle kinetic energy dissipation in the system,

$$
v_{i}^{t+1}=v_{i}+\Delta t \frac{\overrightarrow{\mathbf{f}}_{i}}{m_{\mathbf{y}_{i}}} \text { and } d_{i}^{t+1}=\Delta t v_{i}^{t+1},
$$

where $\Delta t$ is the time step of system evolution (the forward integration step). After the forward integration in each step, the unconstrained $d_{i}^{t+1}$ are added to the current $\mathbf{y}_{i}$ coordinates. Next, a single rigid transformation and a new template pose are obtained by Procrustes analysis [18]. The algorithm converges once the system stabilises, with no template dynamics and entirely dissipated kinetic energy.

Gravitational Approach with Altered Physics [15]. The form of GPE in Eq. (1) includes a reciprocal relation. At the optimal alignment, GPE is limited by $-\infty$. A more robust version of particle dynamics based rigid point set alignment with altered physics laws has been recently proposed [15]. They apply a negative elementwise reciprocal transform to Eq. (1) and obtain a new GPE:

$$
\xi^{-}(\mathbf{E}(\mathbf{R}, \mathbf{t}))=\sum_{i, j} \frac{1}{G m_{\mathbf{y}_{i}} m_{\mathbf{x}_{j}}}\left\|\mathbf{R} \mathbf{y}_{i}+\mathbf{t}-\mathbf{x}_{j}\right\|_{2},
$$

where the transformation $\xi^{-}\left(-\frac{1}{c}\right)=c$ preserves function monotonicity. Note that softening parameter $\epsilon$ is omitted, due to no near-field singularities. With the new form of GPE, the optimal alignment is still achieved when GPE is locally minimal, while there is no reciprocity. Eq. (4) still represents a globally multiply-linked GPE, and the point correspondences are not explicitly encoded in it.

\section{Proof Methodology}

We partially reuse the notations introduced in Sec. 3 and consider the globally multiply-linked gravitational alignment of two point sets, with the reference $\left[\mathbf{x}_{j}\right]=\mathbf{X} \in$ $\mathbb{R}^{D \times N}, j \in\{1, \ldots, N\}$ and the template $\left[\mathbf{y}_{i}\right]=\mathbf{Y} \in$
$\mathbb{R}^{D \times M}, i \in\{1, \ldots, M\} . \quad N$ and $M$ denote the number of points in the reference and template, respectively.

One of the assumptions of GA and BH-RGA is that the optimal alignment corresponds to the state of the locally minimal GPE. We show that this assumption is not fulfiled when optimising for scale $s$ in GPE, i.e., allowing for the extended 7 DoF GPE version of Eq. (4):

$\xi^{-}(\mathbf{E}(\mathbf{R}, \mathbf{t}, s))=\sum_{i, j}\left[\frac{1}{G m_{\mathbf{y}_{i}} m_{\mathbf{x}_{j}}}\right]\left\|\mathbf{R} \mathbf{y}_{i} s+\mathbf{t}-\mathbf{x}_{j}\right\|_{2}$.

Note that it is more convenient to work with the GPE in Eq. (5). Since $G^{-1}$ is a scaling factor and $m_{\mathbf{y}_{i}}$ as well as $m_{\mathbf{x}_{1}}$ are unit masses, we omit the multiplicative term $\left[\frac{1}{G m_{\mathbf{y}_{i}} m_{\mathbf{x}_{j}}}\right]$. Due to the reversibility of $\xi^{-}(\cdot)$, our observations and findings are both valid for the case of GA, Eq. (1) [14] and BH-RGA, Eq. (4) [15].

Analytically, we cannot cover all possible shapes but can work with well formalised parametric shapes such as circles, squares and rectangles, among others. To consider both hollow and volumetric structures, we choose a unit circle $S^{1}$, a unit sphere $S^{2}$, a unit disk in $\mathbb{R}^{2}$ and a unit ball in $\mathbb{R}^{3}$. Our choice falls on these geometric primitives not lastly because many shapes are homeomorphic to one of them. More specifically, we choose both the template and the reference to be simultaneously one of the geometric primitives mentioned above. The template is an exact copy of the reference so that at the optimal alignment, the registration error vanishes. Both point clouds are centred at the origin of the coordinate system. Note that centres of gravity of all examined geometric primitives coincide with the origin of the coordinate system. In our proof, we systematically compare GPE of the optimal alignments and singularities denoted by $\mathbf{E}_{a}$ and $\mathbf{E}_{s}$, respectively. Moreover, we analyse the existence of equienergetic states by deriving the GPE as functions of the template scale.

To summarise - compared to the general discrete setting and assumptions done in GA (see Sec. 3) - we make several further assumptions and contextual simplifications, allowing to apply multivariate calculus in our proof:

- Similarly to [14], we assume that the reference induces the gravitational force field and the template moves in this field until alignment. Interactions between points belonging to the same point set are omitted.

- Without loss of generality, we assume that the translation is resolved in advance. This is a common and widely-used assumption in rigid point set registration $[21,4,10]$. Both points sets are centred at the origin of the coordinate system.

- Since the considered shapes are fully symmetric, the optimal alignment is agnostic to rotations. Moreover, due 
to the shape symmetry, we can avoid the estimation of all individual point-to-point distances and operate with average intra-point distances $\bar{d}$ parametrised by scale instead. In turn, average point distances $\bar{d}$ parametrise GPE in our proof. Based on $\bar{d}$, we find and compare GPE as functions of the alignment configurations parametrised by the scale $s \in[0 ; 1.2]$.

- We partially work in the continuous domain and assume uniform point distribution and mass density.

\section{Hollow Structures in $\mathbb{R}^{2}$ and $\mathbb{R}^{3}$}

In this section, we analyse hollow structures, i.e., a unit circle $S^{1}$ (Sec. 5.1) and a unit sphere $S^{2}$ (Sec. 5.2).

\subsection{Proof for a Unit Circle in $\mathbb{R}^{2}$}

A unit circle $S^{1}$ is defined as a set of all points in 2D which are located at the unit distance to the origin of the coordinate system (the centre of the circle), i.e., all points obeying the equation $x^{2}+y^{2}=1$. We use polar coordinates so that every point of $S^{1}$ is parametrised by the single $\theta$ value (the radius $\rho$ is fixed to 1 ).

Singularity. Suppose, first, that all template points are located in the centre of $S^{1}$. In this case, the distance of every point of the template to every point of the reference $r_{i, j}$ equals to $r$, and $\bar{d}=r$. Correspondingly, the total $\mathbf{E}_{s}$ is equal to

$$
\mathbf{E}_{s}=r N M=N M \text {. }
$$

Optimal Alignment. Suppose the template is registered to the reference. In this case, $r_{i, j}=0$ if and only if points $\mathbf{y}_{i}$ and $\mathbf{x}_{j}$ are in correspondence, and all other combinations result in $r_{i, j} \neq 0$. Next, we find an average $r_{i, j}$ value for all multiply-linked connections.

Without loss of generality - due to the symmetry assume that $\mathbf{x}_{j}$ is always located at $\theta=0$. The distance $d$ of $\mathbf{x}_{0}$ to a point on $S^{1}$ is parametrised by $\theta$. Applying the law of cosines and the sine half-angle identity, we obtain

$$
d=\sqrt{2(1-\cos \theta)}=2 \sin \frac{\theta}{2} .
$$

To obtain the average distance $\bar{d}$, we apply the mean value theorem for the case of a circle resulting in

$$
\bar{d}=\frac{1}{\pi} \int_{0}^{\pi} 2 \sin \frac{\theta}{2} d \theta=-\left.\frac{4}{\pi}\left(\cos \frac{\theta}{2}\right)\right|_{0} ^{\pi}=\frac{4}{\pi} .
$$

Consequently, the GPE of the optimal alignment adds up to

$$
\mathbf{E}_{a}=\bar{d} N M=\frac{4 N M}{\pi} .
$$

Comparing Eqs. (6) and (9), we observe that $\mathbf{E}_{a}>\mathbf{E}_{s}$ by the factor of $\frac{4}{\pi}$. Thus, the state of optimal alignment results

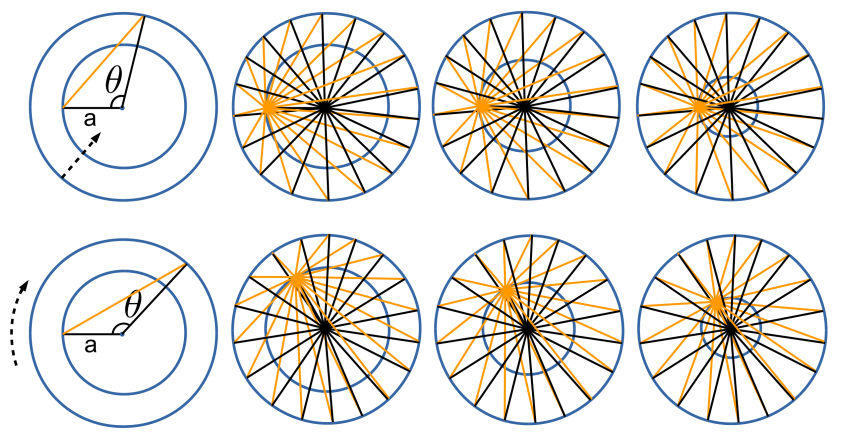

Figure 1: Analysing the average distance $\bar{d}$ as a function of scale $s$ for the unit circle $S^{1}$ in the general case. For every radius of the template circle $a=s, \theta$ parametrises point distances from the template to the reference unit circle (shown in orange).

in $\sim 27 \%$ higher GPE, which is a considerable difference in the energy states. Preliminary, we see that the optimal alignment is not the most optimal state of the particle system. Thus, introducing the scale parameter without further constraints will result in a singularity.

The General Case. To complete and strengthen the analysis of the $\mathbf{E}$ landscape in our test scenario, we would like to find the answers to the following questions: 1) Whether the energy increases monotonically or there are equienergetic states for some values of $s$ between a singularity and the state of optimal alignment, and 2) Whether $\bar{d}=\bar{d}(s)$ is a monotonically increasing function. Consider the case when a template is not aligned with the reference at different $s \in[0 ; 1]$. In this case, we have an additional parameter a, i.e., the radius of the template (see Fig. 1).

Our goal is to derive $\mathbf{E}(a)$ and evaluate it at different $a$ values. Without loss of generality - due to the symmetry - consider that $\mathbf{x}_{j}$ is located at $\theta=0$. Applying the law of cosines and the mean value theorem, we obtain:

$$
\begin{aligned}
& \bar{d}(a)=\frac{1}{\pi} \int_{0}^{\pi} \sqrt{a^{2}+1^{2}-2 a \cos \theta} d \theta= \\
& \begin{cases}\frac{4}{\pi}, & \text { if } a=1, \\
\left.\frac{2|a-1|}{\pi} E\left(\frac{\theta}{2} \mid-\frac{4 a}{(a-1)^{2}}\right)\right|_{\pi} ^{0}, & \text { if } a \neq 1 .\end{cases}
\end{aligned}
$$

with $E(\cdot \cdot \cdot)$ denoting incomplete elliptic integral of the second kind in Legendre form. If $a=1$, Eq. (10) reduces to Eq. (8). Otherwise, it requires an evaluation of $E(\cdot \mid \cdot)$. See Appendix A.1 for more details about elliptic integrals of the second kind in the Legendre form and Appendix A.2 for more details on Eq. (10).

We see that $\mathbf{E}=\mathbf{E}(s)$ is a monotonically increasing function, see Fig. 2 for a plot (in blue), and there are no equienergetic states with the state of the optimal alignment. Generally, there are no equienergetic states with different $s$. 


\subsection{Proof for a Unit Sphere in $\mathbb{R}^{3}$}

Let us consider the unit sphere $S^{2}$ defined as a set of points in 3D which are located at the unit distance to the origin of the coordinate system (the centre of a sphere). Our derivations will coarsely follow the line of derivations for $S^{1}$ in Sec. 5.1.

Singularity. Similarly to the case with $S^{1}$ (see Eq. (6)), the average distance $\bar{d}$ between every template and reference point at a singularity amounts to the sphere radius, and $\mathbf{E}_{s}=N M$.

Optimal Alignment. To find GPE of the optimal point set coincidence, we need to find the average distance $\bar{d}$ from a given point on a surface of the template to every point of the surface of the reference. Since at the optimal alignment, the point sets completely coincide, this problem reduces to the problem of finding an average distance $\bar{d}$ between points on a surface of a sphere.

Suppose, without loss of generality - due to the symmetry - the template point is located on the north pole of the sphere, and $\theta=0$. The distance of this point to any point with the coordinate $(r, \theta, \cdot)$ is $2 \sin (0.5 \theta)$. In total, there are $2 \pi \sin \theta$ points, where $\sin \theta$ is the radius of a circle located at latitude $\theta$ on $S^{2}$. With the help of the mean value theorem, we obtain the expression for $\bar{d}$ :

$$
\begin{gathered}
\bar{d}=\frac{1}{4 \pi} \int_{0}^{\pi} \int_{0}^{2 \pi} 2 \sin \frac{\theta}{2} \sin \theta d \theta d \phi= \\
\int_{0}^{\pi} \sin \frac{\theta}{2} \sin \theta d \theta=\left.\frac{4 \sin ^{3} \frac{\theta}{2}}{3}\right|_{0} ^{\pi}=\frac{4}{3} .
\end{gathered}
$$

Thus, GPE of optimal alignment for $S^{2}$ is $\mathbf{E}_{a}=\bar{d} N M=$ $\frac{4 N M}{3}$.

The General Case. We affirm that there are no equienergetic states with the same energy values as $\mathbf{E}(0)$. We parametrise the template by the radius $a \in[0 ; 1.2]$, and derive the expression of $\bar{d}(a)$ for the general case:

$$
\begin{aligned}
& \bar{d}(a)=\frac{1}{4 \pi} \int_{0}^{\pi} \int_{0}^{2 \pi} \sqrt{a^{2}+1-2 a \cos \theta} \sin \theta d \theta d \phi= \\
& =\left\{\begin{array}{ll}
\frac{1}{2} \int_{0}^{\pi} \sin \theta d \theta, & \text { if } a=0, \\
\frac{1}{2} \int_{0}^{\pi} \sqrt{a^{2}+1-2 a \cos \theta} \sin \theta d \theta, & \text { if } a>0 .
\end{array}=\right. \\
& = \begin{cases}1, & \text { if } a=0, \\
\left.\frac{\left(a^{2}+1-2 a \cos \theta\right)^{\frac{3}{2}}}{6 a}\right|_{0} ^{\pi}, & \text { if } a>0 .\end{cases}
\end{aligned}
$$

Eq. (12) can be evaluated for different $a$ in closed form. See Appendix A.3 for more details on Eq. (12). If $a=1$, Eq. (12) evaluates to $\frac{4}{3}$. The values of the integral for $a \in$ $[0 ; 1.2]$ are plotted in Fig. 2 (in green).

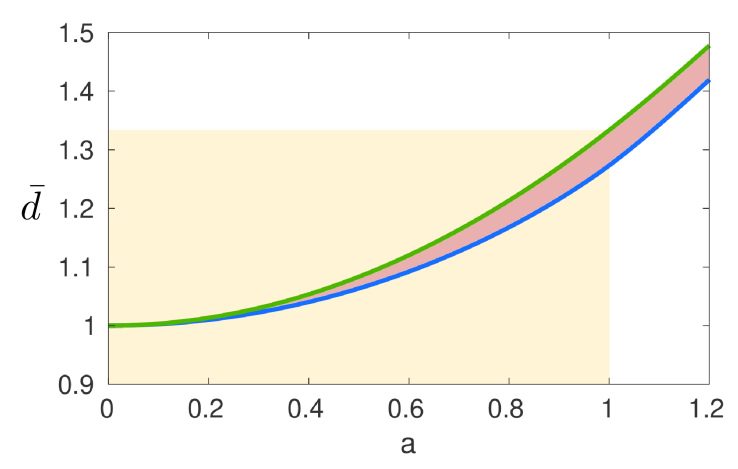

Figure 2: Average distances $\bar{d}(a)$ as the functions of the template radius $a$, for $S^{1}$ (blue graph) and $S^{2}$ (green graph).

Similarly to $S^{1}$, a singularity is an energetically the most stable state in the case of $S^{2}$. Compared to $S^{1}$, the difference in $\bar{d}$ increases to $\sim 33 \%$.

\section{Volumetric Structures in $\mathbb{R}^{2}$ and $\mathbb{R}^{3}$}

Next, we are considering volumetric structures with uniform density, i.e., a unit disk in $\mathbb{R}^{2}$ and a unit ball in $\mathbb{R}^{3}$. When deriving the average distances between points in volumetric structures in polar and spherical coordinates, we need to compensate for non-uniform volume sampling caused by monotonically increasing volume encompassed between infinitesimally increasing coordinates $r$ and $r+d r$, $\theta$ and $\theta+d \theta$ and $\phi$ and $\phi+d \phi$. The probability $p_{r}$ to find a point inside a sphere of radius $r<a$ when points are uniformly sampled in a ball of radius $a$ is

$$
p_{a}=\left(\frac{r}{a}\right)^{n}
$$

where $n$ is the space dimensionality [17]. The probability density function of $r$ is the derivative of $p_{a}$ with respect to $r$ :

$$
\frac{d}{d r} p_{a}=\left(\frac{n r^{n-1}}{a^{n}}\right)
$$

We use Eq. (14) in Secs. 6.1 and 6.2 for uniform space sampling in a unit disk and a unit sphere.

\subsection{Proof for a Unit Disk in $\mathbb{R}^{2}$}

A unit disk in $\mathbb{R}^{2}$ is a set of all points enclosed by $S^{1}$. We assume that the points inside the unit disk are sampled uniformly. Recall that uniform sampling in polar coordinates does not result in uniform space sampling, and we apply Eq. (14) to compensate for the inhomogeneous sampling. Thus, the probability $p_{a}$ to find a point sampled from a uniform distribution inside a circle of radius $q \leq a$ is $P(p \leq q)=\left(\frac{r}{a}\right)^{2}$, and $2 r$ is the probability density function of $p_{a}$ in 2D according to Eq. (14). 
Singularity. Consider a singularity, i.e., the state when the whole volume of the template collapses into a single point. For the volumetric singularity in $2 \mathrm{D}$, we obtain the following expression for the average distance $\bar{d}$ from the disk centre to uniformly sampled points inside the disk:

$$
\bar{d}=\frac{1}{\pi} \int_{0}^{1} 2 r \int_{0}^{\pi} r d \theta d r=\int_{0}^{1} 2 r^{2} d r=\frac{2}{3} .
$$

Optimal Alignment. For the aligned volumetric data in 2D, we consider the average distance between a point on a circle of radius $r \leq 1$ and a circle of radius $q \leq 1$ inside a unit disk:

$$
\begin{aligned}
& \bar{d}=\frac{1}{\pi} \int_{0}^{1} 2 r \int_{0}^{1} 2 q \int_{0}^{\pi} \sqrt{r^{2}+q^{2}-2 r q \cos \theta} d \theta d q d r= \\
& \left.\frac{8}{\pi} \int_{0}^{1} r \int_{0}^{1} q|q-r| E\left(\frac{\theta}{2} \mid-\frac{4 r q}{(q-r)^{2}}\right)\right|_{\pi} ^{0} d q d r \approx 0.9062 .
\end{aligned}
$$

We solve Eq. (16) by applying the composite trapezoidal rule of numerical integration with the step size $\tau=10^{-2}$. The obtained value $\bar{d}=0.9062$ agrees with the average analytical distance derived by integrating over the probability density of the distances between points in a disk [12]:

$$
\bar{d}=\frac{125}{45 \pi} \approx 0.9054
$$

We see that for a unit disk in $\mathbb{R}^{2}$, the optimal alignment results in $\sim 35.8 \%$ higher GPE compared to a singularity. The General Case. We derive the average distances for the case $a \in[0 ; 1.2]$ in a similar fashion as Eq. (16) while taking into account the parametric upper limit of integration for $q$ and the parametric derivative of the probability to find a point within a disk of radius $a$ :

$\bar{d}(a)=\left.\frac{8}{\pi} \int_{0}^{1} r \int_{0}^{a} \frac{q}{a^{2}}|q-r| E\left(\frac{\theta}{2} \mid-\frac{4 r q}{(q-r)^{2}}\right)\right|_{\pi} ^{0} d q d r$.

See more details on Eq. (18) in Appendix A.4. The function $\bar{d}=\bar{d}(a)$ for the unit disk in $\mathbb{R}^{2}$ is plotted in Fig. 4 (in blue).

\subsection{Proof for a Unit Ball in $\mathbb{R}^{3}$}

A unit ball in $\mathbb{R}^{3}$ is a set of points enclosed by $S^{2}$. We consider that the space inside the unit ball is sampled uniformly. Recall that we use spherical coordinates, and uniform sampling of the coordinates does not result in the uniform space sampling. Thus, to find the average distances between uniformly sampled points in a unit sphere, we have to introduce a sampling density factor. The probability of finding a point sampled from a uniform distribution, inside a sphere of radius $q \leq a$, is $P(p \leq q)=\left(\frac{r}{a}\right)^{3}$, where $a$ is a radius of a sphere. For a unit ball, the rate of the probability change is the derivative of the density with respect to the radius, i.e., $\frac{d}{d r} r^{3}=3 r^{2}$. We use this rate of change to find a point in a ball of radius $r$ in further derivations.

Singularity. Suppose all points of the template collapse to the centre of gravity of the reference, and all other points are uniformly sampled inside the reference. The mean distance between the centre of the unit ball and spheric shell of radius $r$ is $r \frac{\sin \theta}{2}$, and

$$
\bar{d}=\int_{0}^{1} 3 r^{2} \int_{0}^{\pi} r \frac{\sin \theta}{2} d \theta d r=\int_{0}^{1} 3 r^{3} d r=\frac{3}{4} .
$$

Optimal Alignment. Next, consider the state of optimal alignment of two unit balls. In this case, we require two rates of probability changes for finding a point in a unit ball of radii $0 \leq r \leq 1$ and $0 \leq q \leq 1$. Following Eq. (14), we see that those are equal to $3 r^{2}$ and $3 q^{2}$, respectively. Thus, the mean distance between two uniformly sampled points inside a unit ball can be calculated as

$$
\begin{aligned}
& \bar{d}= \\
& \int_{0}^{1} 3 r^{2} \int_{0}^{1} 3 q^{2} \int_{0}^{\pi} \frac{\sqrt{r^{2}+q^{2}-2 r q \cos \theta} \sin \theta}{2} d \theta d q d r .
\end{aligned}
$$

First, consider the inner integral over $\theta$ :

$$
\begin{aligned}
\int_{0}^{\pi} \frac{\sqrt{r^{2}+q^{2}-2 r q \cos \theta} \sin \theta}{2} d \theta \stackrel{(r, q \geq 0)}{\underline{=}} \\
\frac{(r+q)^{3}-(r-q)^{2}|r-q|}{6 r q} \stackrel{(r \geqq q)}{=} \frac{6 r^{2} q+2 q^{3}}{6 r q} .
\end{aligned}
$$

We analyse the cases 1) $r \geq 0$ and $q \geq 0$ and 2) $r \geq q$ separately. This allows us to get rid of the modulo operator while exploiting the symmetry at evaluating Eq. (20), see Fig. 3-(a) for the visualisation. Assuming $r \geq q$, we represent the total integral as twice the integral which accounts for the condition $r \geq q$ :

$$
\bar{d}=2 \int_{0}^{1} \int_{0}^{1} \frac{9 q^{2} r^{2}\left(6 r^{2} q+2 q^{3}\right)}{6 r q} d q d r=\frac{36}{35} .
$$

We observe that the energy of the optimal alignment in the case of the unit ball is larger than the energy of a singularity by $\sim 37 \%$.

The General Case. Finally, we generalise the energy to all cases between a singularity $(s=0)$ and the optimal alignment $(s=1)$. We calculate the average distance between points sampled uniformly from a ball of radius $a \in[0 ; 1.2]$ (modelling the transformed template) and points sampled uniformly from a unit ball (modelling the reference).

Compared to the optimal alignment, there are several modifications in the calculation of $\bar{d}$. First, the rate of change of the probability to find a point in a ball of radius $a$ is $\frac{3 q^{2}}{a^{3}}$. Second, the integral over $d q$ is evaluated in the interval $[0 ; a]$. Moreover, we cannot take advantage of the 
(a)

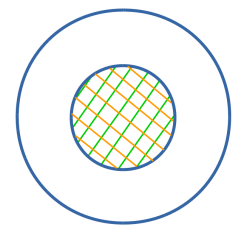

(b)

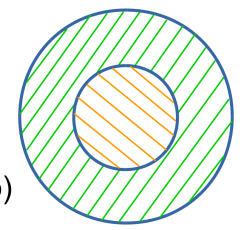

Figure 3: (a): The symmetric case when a point of the template and the reference are sampled from the same volume. (b): If the template and the reference differ, points are sampled from non-intersecting subvolumes.

full symmetry as it is the case in Eq. (22). For the general case, Eq. (20) modifies to

$$
\begin{aligned}
& \bar{d}(a)= \\
& \int_{0}^{1} 3 r^{2} \int_{0}^{a} \frac{3 q^{2}}{a^{3}} \int_{0}^{\pi} \frac{\sqrt{r^{2}+q^{2}-2 r q \cos \theta} \sin \theta}{2} d \theta d q d r .
\end{aligned}
$$

Under the condition that $r \geq q$, the inner integral over $\theta$ evaluates as in Eq. (21). In contrast to Eq. (22), we can exploit the partial symmetry. Thus, we represent Eq. (23) as a sum of two integrals covering the case when 1) $r \leq a$ and $q \leq a$ and 2) $q \in[0 ; a]$ while $r \in[a ; 1]$ :

$$
\bar{d}(a)=d_{a}(a)+d_{a, 1}(a) .
$$

The first case covers all point pairs drawn from the unit balls of all radii not greater than $a$, see Fig. 3-(a). The second case covers all point pairs so that one point is drawn from the hollow unit ball (the volume circumscribed by a sphere with $r \leq a$ is not considered, the ball of radius $a$ is removed), and the ball of radius $a$, see Fig. 3-(b). In case 1), we can use the full symmetry inside the ball of radius $a$ to guarantee that $r \geq q$. Following the similar principles as in the case of optimal alignment in Eq. (22), we obtain:

$$
d_{a}(a)=2 \int_{0}^{a} \int_{0}^{a} \frac{9 q^{2} r^{2}}{a^{3}} \frac{\left(6 r^{2} q+2 q^{3}\right)}{6 r q} d q d r=\frac{36}{35} a^{4} .
$$

In case 2), the integration intervals do not intersect, and the condition $r \leq q$ is satisfied. Likewise, we can use the simplified expression without a modulo operator from Eq. (21) to calculate $d_{a, 1}$ :

$$
\begin{aligned}
& d_{a, 1}(a)=\int_{a}^{1} \int_{0}^{a} \frac{3 r q}{2 a^{3}}\left(6 r^{2} q+2 q^{3}\right) d q d r= \\
& \int_{a}^{1} \frac{3}{5} r\left(5 r^{2}+a^{2}\right) d r=-\frac{21 a^{4}-6 a^{2}-15}{20} .
\end{aligned}
$$

By merging Eqs. (25)-(26) we obtain the final $\bar{d}(a)$ :

$\bar{d}(a)=\frac{36}{35} a^{4}-\frac{21 a^{4}-6 a^{2}-15}{20}=\frac{26 \frac{1}{4}+10 \frac{1}{2} a^{2}-\frac{3}{4} a^{4}}{35}$.

Eq. (27) agrees with Eqs. (19) and (22) for the cases of a volumetric singularity and the optimal volumetric alignment, respectively, i.e., $\bar{d}(0)=\frac{3}{4}$, and $\bar{d}(1)=\frac{36}{35}$. The

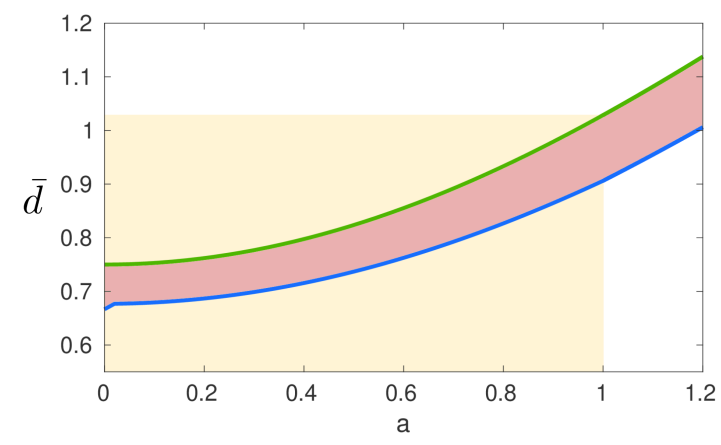

Figure 4: Average distances $\bar{d}(a)$ as the functions of the template radius $a$, for a unit disk in $\mathbb{R}^{2}$ (blue graph) and a unit ball in $\mathbb{R}^{3}$ (green graph).

function $\bar{d}=\bar{d}(a)$ for the unit ball in $\mathbb{R}^{3}$ is plotted in Fig. 4 (in green).

\section{Discussion and Experimental Validation}

In Secs. 5.1-6.2 we show that GPE of a singularity is always smaller than GPE of the optimal alignment under globally multiply-linked point interactions. The gap between the energy values of the optimal alignment and singularities is slightly larger for the hollow geometric primitives ( $S^{1}$ and $S^{2}$ ) compared to the volumetric geometric primitives (a unit disk and a unit ball). The gap is larger for 3D structures (both for hollow and volumetric geometric primitives). Thus, we have analytically shown that a singularity is an energetically more favourable state if the alignment is performed as proposed in $[14,15]$ with the scale parameter.

In addition, we perform multiple experiments with various shapes (human bodies, animals and 3D surfaces) with scale optimisation. We select one point cloud (Stanford bunny [6]) and create multiple initial misalignments by systematically rotating the template. In total, we obtain 500 initial misalignments by changing the angles around the $x$-, $y$ - and $z$-axes with the angular step size of $\frac{\pi}{5}$ radians. All alignments result in a singularity. Fig. 5-(a) showcases one registration result with the bunny [6]. The template collapses to the centre of gravity of the reference.

So far, we have not found a configuration which is a nonsingularity and which has a lower GPE than the GPE of a singularity. As mentioned earlier, many real-world shapes are homeomorphic to a circle or a sphere which are in focus of our analysis.

One way to enable scale resolution by rigid gravitational methods is to restrict point interactions to local neighbourhoods or use prior matches, see Fig. 5-(b), -(c). In Fig. 5-(b), we restrict interactions so that every template point interacts with 30 reference points on average. Both input point clouds differ by the factor 1.2 in scaling. For the successful scale resolution, interaction volume of every template point has to encompass the correct corresponding point of 


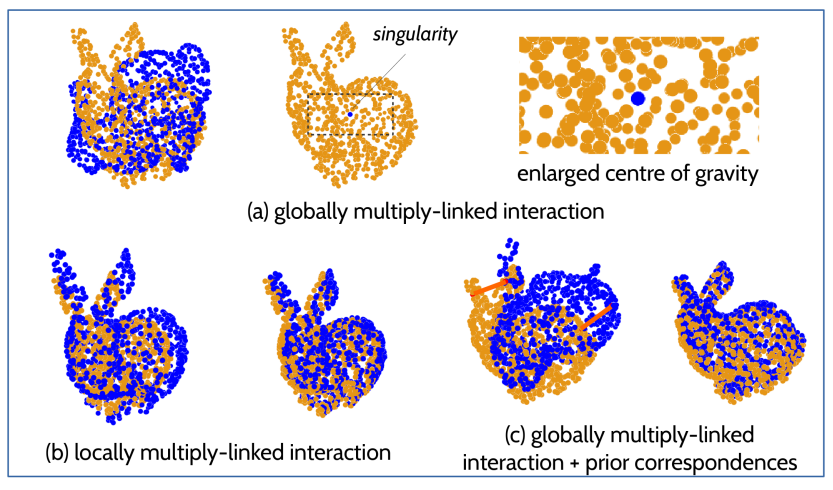

Figure 5: If template scaling is allowed, globally multiply-linked gravitational point set alignment results in a singularity (a). To remedy scaling resolution, point interactions can be restricted to local neighbourhoods (b) or prior matches with high masses can be embedded (c).

the reference. In Fig. 5-(c), all points interact in a globally multiply-linked manner, and we embed two prior matches. In the case of prior matches, only corresponding points interact and their masses are orders of magnitude larger than the default masses. As a result, scale and rotation are resolved, and a singularity is avoided. Since point masses in GA can be set differently, points which are more likely to correspond can be assigned higher masses in advance.

\section{Conclusion}

We conclude that in rigid globally multiply-linked gravitational point set alignment, allowing for the scale optimisation leads to a singularity, i.e., shrinkage of the template to the centre of gravity of the reference. We prove for the regular geometric shapes in 2D and 3D that GPE of a singularity is always smaller than GPE of the optimal alignment. We do not find equienergetic states between optimal alignments and singularities, as the GPE parametrised by the average intra-point distances is a monotonically increasing function. For practical applications, this means that it is highly unlikely that multiply-linked gravitational point set alignment can resolve the scale. Our experimental validations with point sets of various shapes and sizes all have resulted in singularities when aligned in a multiply-linked manner and allowing for scaling.

We hope that our analysis will help in the development of robust point set alignment approaches relying on particle dynamics of the next generation and inspire new directions. In Sec. 7, we have mentioned some scenarios where scale resolution with particle dynamics is better posed. In future work, we will systematically analyse locally-multiply linked policies and prior matches in GA. Moreover, we plan to study how deviations from a circle or a sphere affect the GPE landscape and the gaps between GPE of singularities and optimal alignments.

\section{A. Appendix}

\section{A.1. Elliptic Integrals}

Elliptic integrals of the second kind in the Legendre form are integrals of type

$$
E(\phi, k)=\int_{0}^{\phi} \sqrt{1-k \sin ^{2} \theta} d \theta
$$

Initially, elliptic integrals arise in the study of the arc length problem for ellipses. Elliptic integrals, as a rule, cannot be simplified and analytically evaluated. There are multiple methods available for numerical calculation $[5,11]$.

\section{A.2. Equivalency of Integrals in Eq. (10)}

We show that both integrals in Eq. (10) are equivalent:

$$
\begin{aligned}
& \int \sqrt{a^{2}+1-2 a \cos \theta} d \theta=\int \sqrt{a^{2}+1+\frac{4 a(1-\cos \theta)}{2}-2 a} d \theta= \\
& \int \sqrt{4 a \sin ^{2} \frac{\theta}{2}+\frac{(a-1)^{4}}{(a-1)^{2}}} d \theta=\sqrt{(a-1)^{2}} \int \sqrt{\frac{4 a \sin ^{2} \frac{\theta}{2}}{(a-1)^{2}}+1} d \theta= \\
& 2|a-1| E\left(\frac{\theta}{2},-\frac{4 a}{(a-1)^{2}}\right) .
\end{aligned}
$$

When $a=1$, Eq. (29) cannot be analytically evaluated because of the elliptic integral. At the same time, we see that

$$
\lim _{a \rightarrow 1} \frac{1}{\pi} \int_{0}^{\pi} \sqrt{a^{2}+1^{2}-2 a \cos \theta} d \theta=\frac{4}{\pi} .
$$

\section{A.3. The General Case for $\boldsymbol{S}^{\mathbf{2}}$ (Analysis of Eq. (12))}

If $a=0$ is directly substituted into the left side of Eq. (12), we obtain $\frac{1}{2} \int_{0}^{\pi} \sin \theta d \theta=1$. We also see that

$$
\lim _{a \rightarrow 0} \frac{1}{2} \int_{0}^{\pi} \sqrt{1+a^{2}+2 a \cos \theta} \sin \theta d \theta=1 .
$$

Due to the $6 a$ divisor in Eq. (12), the function cannot be evaluated for $a=0$.

\section{A.4. The Discretisation of Eq. (18)}

Eq. (18) can be evaluated numerically by applying the composite trapezoidal rule of numerical integration:

$$
\left.\bar{d} \approx \frac{8 a}{\pi n m} \sum_{r=0}^{1} r \sum_{q=0}^{a} \frac{q}{a^{2}}|q-r| E\left(\frac{\theta}{2} \mid-\frac{4 r q}{(q-r)^{2}}\right)\right|_{\pi} ^{0},
$$

where $n=\tau^{-1}$ and $m=a \tau^{-1}$ are the numbers of intervals for $r$ and $q$, respectively, with the step size $\tau=10^{-2}$ (both $n$ and $m$ are chosen as ratios of the respective integration regions to $\tau$ ). 


\section{References}

[1] S. J. Aarseth. Gravitational N-Body Simulations: Tools and Algorithms. Cambridge Monographs on Mathematical Physics. Cambridge University Press, 2003. 3

[2] S. Agarwal and B. Bhowmick. 3d point cloud registration with shape constraint. In International Conference on Image Processing (ICIP), pages 2199-2203, 2017. 1, 2

[3] S. A. Ali, V. Golyanik, and D. Stricker. Nrga: Gravitational approach for non-rigid point set registration. International Conference on $3 D$ Vision (3DV), pages 756-765, 2018. 1, 2

[4] P. J. Besl and N. D. McKay. A method for registration of 3-d shapes. Transactions on Pattern Analysis and Machine Intelligence, 14(2):239-256, 1992. 1, 2, 3

[5] R. Bulirsch. Numerical calculation of elliptic integrals and elliptic functions. Numerische Mathematik, 7(1):78-90, 1965. 8

[6] The Stanford 3D Scanning Repository. http:// graphics.stanford. edu/data/3Dscanrep/. 7

[7] Y. Chen and G. Medioni. Object modelling by registration of multiple range images. Image and Vision Computing (IVC), 10(3): 145 - 155, 1992. 2

[8] H. Chui and A. Rangarajan. A new point matching algorithm for non-rigid registration. Computer Vision and Image Understanding (CVIU), 89(2-3):114-141, 2003. 2

[9] Y. Deng, A. Rangarajan, S. Eisenschenk, and B. C. Vemuri. A riemannian framework for matching point clouds represented by the schrödinger distance transform. In Computer Vision and Pattern Recognition (CVPR), pages 3756-3761, 2014. 2

[10] A. Fitzgibbon. Robust registration of $2 \mathrm{~d}$ and $3 \mathrm{~d}$ point sets. Image and Vision Computing (IVC), 21:1145-1153, 2002. 2, 3

[11] T. Fukushima. Fast computation of complete elliptic integrals and jacobian elliptic functions. Celestial Mechanics and Dynamical Astronomy, 105(4), 2009. 8

[12] R. García-Pelayo. Distribution of distance in the spheroid. Journal of Physics A: Mathematical and General, 38(16):3475-3482, 2005. 6

[13] S. Gold, A. Rangarajan, C.-P. Lu, S. Pappu, and E. Mjolsness. New algorithms for $2 \mathrm{~d}$ and $3 \mathrm{~d}$ point matching: Pose estimation and correspondence. Pattern Recognition, 31:1019-1031, 1999. 2

[14] V. Golyanik, S. A. Ali, and D. Stricker. Gravitational approach for point set registration. In Computer Vision and Pattern Recognition (CVPR), pages 5802-5810, 2016. 1, 2, 3, 7

[15] V. Golyanik, C. Theobalt, and D. Stricker. Accelerated gravitational point set alignment with altered physical laws. In International Conference on Computer Vision (ICCV), 2019. $1,2,3,7$

[16] M. Greenspan and G. Godin. A nearest neighbor method for efficient icp. In International Conference on 3-D Digital Imaging and Modeling (3DIM), pages 161-168, 2001. 2

[17] R. Harman and V. Lacko. On decompositional algorithms for uniform sampling from n-spheres and n-balls. Journal of Multivariate Analysis, 101(10):2297 - 2304, 2010. 5
[18] B. K. P. Horn, H. M. Hilden, and S. Negahdaripour. Closedform solution of absolute orientation using orthonormal matrices. Journal of the Optical Society of America A, 5(7):1127-1135, 1988. 3

[19] P. Jauer, I. Kuhlemann, R. Bruder, A. Schweikard, and F. Ernst. Efficient registration of high-resolution feature enhanced point clouds. Transactions on Pattern Analysis and Machine Intelligence (TPAMI), 41(5):1102-1115, 2019. 2

[20] B. Jian and B. Vemuri. Robust point set registration using gaussian mixture models. Transactions on Pattern Analysis and Machine Intelligence (TPAMI), 33(8):1633-1645, 2011. 1,2

[21] A. Myronenko and X. Song. Point set registration: Coherent point drift. Transactions on Pattern Analysis and Machine Intelligence (TPAMI), 32(12):2262-2275, 2010. 1, 2, 3

[22] S. Rusinkiewicz and M. Levoy. Efficient variants of the icp algorithm. In International Conference on 3-D Digital Imaging and Modeling (3DIM), 2001. 2

[23] S. Shimada, V. Golyanik, E. Tretschk, D. Stricker, and C. Theobalt. DispVoxNets: Non-rigid point set alignment with supervised learning proxies. In International Conference on $3 D$ Vision (3DV), 2019. 2

[24] Y. Tsin and T. Kanade. A correlation-based approach to robust point set registration. In European Conference on Computer Vision (ECCV), pages 558-569, 2004. 2

[25] Z. Zhou, J. Tu, C. Geng, J. Hu, B. Tong, J. Ji, and Y. Dai. Accurate and robust non-rigid point set registration using students-t mixture model with prior probability modeling. Scientific Reports, 8, 2018. 2 